\title{
Alveolar echinococcosis of the liver: percutaneous stent therapy in Budd-Chiari syndrome
}

\author{
J Vogel, J Görich, E Kramme, E Merkle, R Sokiranski, P Kern, H J Brambs
}

\begin{abstract}
Background and Aims-Infiltration of the hepatic veins in alveolar echinococcosis can lead to the development of the BuddChiari syndrome. The medical and surgical treatment of this condition is generally unsatisfactory. The results of successful interventional treatment with percutaneous stent implantation in the hepatic veins are reported.

Methods-Using a transjugular approach, metal mesh stents (Boston Scientific, Medi-Tech Accuflex $8 / 60 \mathrm{~mm}$ ) were placed in the median and left hepatic veins of a 53 year old woman. After the intervention, oral chemotherapy with albendazole ( $2 \times 400 \mathrm{mg} /$ day) was continued, but no anticoagulants were given.

Results-Stent placement was performed without complications. The clinical picture improved rapidly: normalisation of portal blood flow was confirmed by Doppler ultrasound and there was improvement of liver function, reduction of oesophageal varices, and disappearance of ascites. A follow up examination at 15 months showed no evidence of stent occlusion.

Conclusions-Treatment of portal hypertension in alveolar echinococcosis of the liver is problematic. In selected patients with portal hypertension secondary to hepatic vein stenoses but no cirrhosis, percutaneous stent placement in the hepatic veins represents a promising treatment alternative.

(Gut 1996; 39: 762-764)
\end{abstract}

Department of

Radiology, University

Clinic of Ulm,

Germany

J Vogel

J Görich

E Merkle

R Sokiranski

H J Brambs

Infectious Diseases Section, University Clinic of Ulm, Germany E Kramme P Kern

Correspondence to: Dr Jochen Vogel, Universitätsklinik Ulm, Radiologie I, Robert Koch Strasse, D-89081 Ulm, Germany.

Accepted for publication 20 June 1996
Keywords: alveolar echinococcosis, stent, Budd-Chiari syndrome.

The ten year survival rates for alveolar echinococcosis of the liver have increased from $10 \%$ in the 1960 s to about $85 \%$ today. ${ }^{1}$ This is due both to improved surgical and medical treatments concepts ${ }^{12}$ and to early diagnosis using serological methods and radiological imaging techniques. A complete cure, however, can only be achieved in those few patients in which radical surgical resection is feasible. Systemic chemotherapy with albendazole or mebendazole does not usually lead to complete extermination of the larvae, but often prevents further spread of the disease. ${ }^{2}$ The development of portal hypertension with ascites is cited in the literature as a frequent complication. This is caused by biliary cirrhosis secondary to infestation of the bile ducts or to stenosis of the portal or hepatic veins in the Budd-Chiari syndrome. In such cases, the creation of a portocaval shunt or surgical resection are usually considered. This is the report of a patient in whom the development of portal hypertension was caused by a dorsally situated focal infestation of the liver with increasing stenosis of the hepatic veins, which we treated with the percutaneous placement of metal mesh stents in the median and left hepatic veins.

\section{Case report}

A 53 year old white woman had alveolar echinococcosis of the liver with infiltrative growth, encasement of the inferior vena cava, and retroperitoneal spread to the adrenal glands and diaphragm was first diagnosed in 1986. After exploratory laparotomy in 1986, plication of the diaphragm was performed in 1987. In the next years, a slow but steady progression of the disease under often interrupted administration of mebendazole (beginning in 1993, albendazole) was noted. In 1993, a pleural effusion accompanied the recurrence of transdiaphragmatic infestation with pleural infiltration, requiring parietal pleurectomy. In 1994, ascites developed and, despite administration of aldactone $(200 \mathrm{mg}$ four times daily) furosemide $(80 \mathrm{mg}$ four times daily) ultimately required weekly paracentesis.

On admission to hospital in Spring 1995, an abdominal circumference of $96 \mathrm{~cm}$ secondary to massive ascites was measured. Endoscopy disclosed evidence of portal hypertension, such as grade II oesophageal varices reaching to the gastric fundus. Neither Doppler sonography nor angiography (Fig 1B) demonstrated blood flow in the portal vein. Demonstration by computed tomography of a large, dorsally situated focus of infestation impinging on the hepatic veins and the significantly delayed uptake of contrast medium in the hepatic parenchyma (Fig 1A) provided evidence of stenosis in the hepatic veins. The stenoses of the median and left hepatic veins was subsequently confirmed by transjugular phlebography of the hepatic veins (Fig 1C) and verified by the measurement of a prestenotic to poststenotic venous pressure difference of $23 \mathrm{~cm} \mathrm{H}_{2} \mathrm{O}$. The right hepatic vein was completely occluded. 

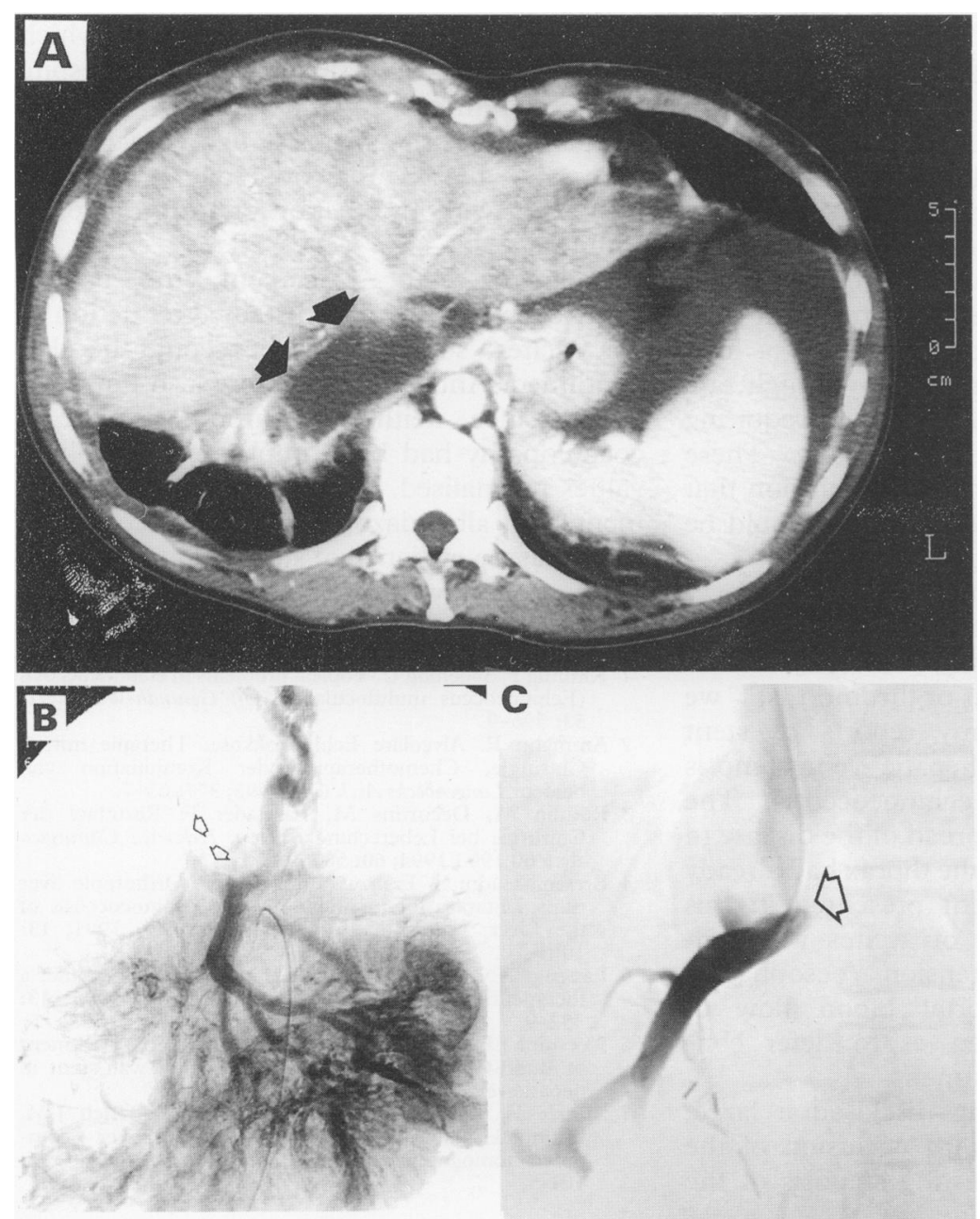

Figure 1: Alveolar echinococcosis of the liver with posterior localisation and Budd-Chiari syndrome. (A) CT: large, non-homogeneous, hypodense focus with calcifications on the dorsal aspect of the liver adjacent to the orifice of the hepatic veins (arrow). There is occlusion of the retrohepatic segment of the inferior vena cava with collateralisation of the azygos vein. Uptake of contrast medium in the hepatic parenchyma is delayed and nonhomogenous (Budd-Chiari syndrome). (B) Mesentericography, venous phase: weak contrast of the portal vein (arrows) with concommitant prominent collateralisation of the vena coronaria ventriculic. (C) Retrograde transjugular phlebography of the hepatic veins: high grade stenosis of the left and median hepatic veins at their orifice into the preatrial segment of the inferior vena cava with jet phenomenon (arrow). The right hepatic vein is occluded.

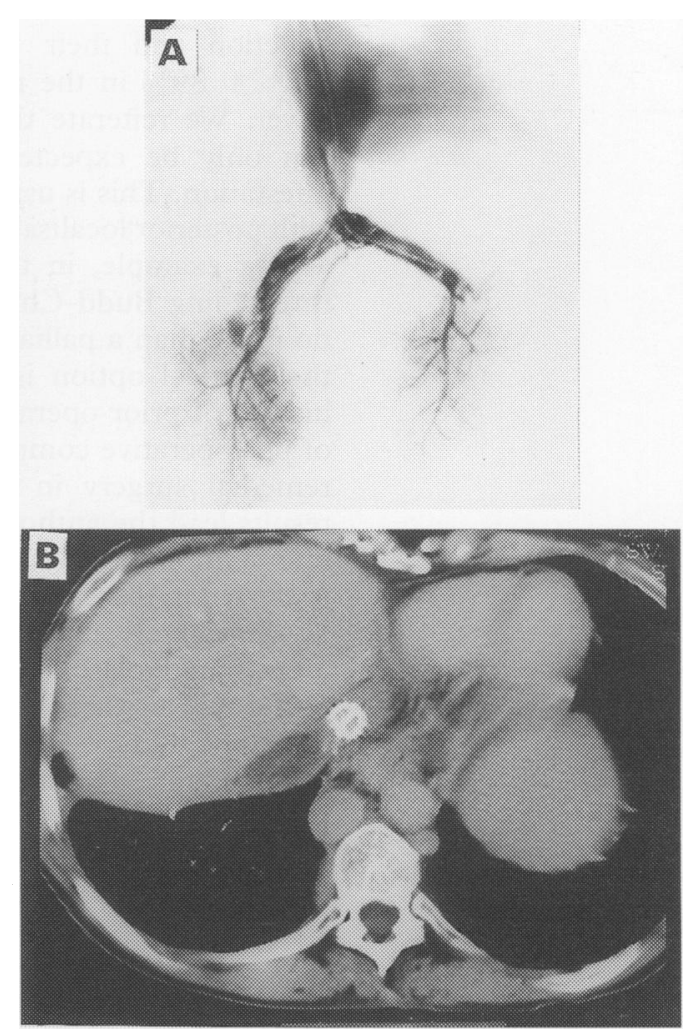

Figure 2: After transjugular stent placement in the left and median hepatic veins. (A) Retrograde, transjugular phlebography of the hepatic veins: strong, unhindered flow of contrast medium. (B) CT: both stents lie parallel in the inferior vena cava, bypass the echinococcus focus and appear homogeneous after intravenous application of contrast medium.

increased to $40 \mathrm{~cm} / \mathrm{s}$. At a follow up examination one week later, abdominal circumference had decreased to $84 \mathrm{~cm}$ and ultrasound showed only discrete remaining ascites.

After a follow up period of 15 months, the patient feels subjectively well. Both Doppler ultrasound and computed tomography continued to show stent patency and there was no discernible ascites. Prothrombin time (Quick) has increased from $48 \%$ to $97 \%$ and endoscopy shows only grade I oesophageal varices.

\section{Discussion}

Radical surgery is followed by cure in only $20 \%-40 \%$ of patients with asymptomatic alveolar echinococcosis. ${ }^{2}$ Thus surgical procedures are often no more than palliative in nature and an additional, lifelong chemotherapy is required. ${ }^{2}$ Moreover, postoperative morbidity rates between $14 \%$ and $53 \%$ have been reported. ${ }^{3} \mathrm{~A}$ study by Bresson-Hadni ${ }^{4}$ reported the results of 17 orthotopic liver transplants in patients with alveolar echinococcosis of the liver. Fifteen of these patients exhibited portal hypertension, which in seven patients was secondary to the Budd-Chiari syndrome with posterior localisation. The remaining four patients had either disease spread to the liver hilus with infiltration of the portal vein or biliary cirrhosis. A second operation was required in a significantly higher proportion of patients with alveolar ecchinococcosis receiving orthotopic liver transplants than in a comparable group without cestode Accuflex $8 / 60 \mathrm{~mm}$ ) in the median an hepatic veins (Fig $2 \mathrm{~A}$ ).

Immediately after placement of the stent, Doppler sonography showed an appreciable increase in portal flow $(21 \mathrm{~cm} / \mathrm{sec})$, which later 
infection and their 15 month survival rate $(75 \% v 89 \%$ in the non-infected group) was lower. We reiterate that a curative operation can only be expected in cases of localised infestation. This is usually not seen in patients with posterior localisation, such that operation, as, for example, in the treatment of the life threatening Budd-Chiari syndrome, often has no more than a palliative character. As a rule, the surgical option is confounded by a long history of prior operations and by a high rate of postoperative complications often requiring remedial surgery in their own right. These results lead the authors to the conclusion that interventional radiological methods should be preferred in these patients.

While interventional methods (stent placement, angioplasty, thrombolysis) are often performed in cases of Budd-Chiari syndrome due to membrane stenosis or thrombosis, ${ }^{5}{ }^{6}$ we were unable to find any reports of stent placement in cases of hepatic vein stenosis secondary to alveolar echinococcosis. The diagnosis of infiltrating spread of the disease to adjacent organs and into the thorax had already been made in the patient presented in this report. The appearance of ascites with evidence of portal hypertension (oesophageal varices, absence of portal blood flow at Doppler sonography) led us to defer consideration of surgical treatment.

Computed tomography disclosed a large, dorsally situated focus with occlusion of the inferior vena cava, suggesting stenosis of the hepatic veins (Budd-Chiari syndrome), which was later confirmed angiographically. Despite a reduction in liver function, the high pressure gradient across the stenosis augured well for a successful therapeutic outcome after stent placement without creation of an intrahepatic shunt.

Following stent placement, the patient experienced a rapid and sustained reduction of her ascites, so that diuretics could be discontinued. Anticoagulants were also not indicated, since neither a thrombosis nor a coagulopathy had been implicated. The liver values normalised. With the continued treatment with albendazole ( $400 \mathrm{mg}$ twice daily), there was no evidence of stent occlusion in the 15 month follow up period.

1 Kimmig P, Schelling U. Topical problems in echinococcosis (Echinococcus multilocularis). Öff Gesundh-Wes 1991 53: 596-9.

2 Ammann R. Alveoläre Echinokokkose: Therapie mittels Chirurgie, Chemotherapie oder Kombination von beiden? Langenbecks Arch Chir 1992; 377: 65-7.

3 Röthlin M, Decurtins M, Largiader F. Resultate der Chirurgie bei Leberechinokokkose. Helvetica Chirurgica Acta 60 1993/1994; 60: 587-92.

4 Bresson-Hadni S, Franza A, Miguet JP. Orthotopic liver transplantation for incurable alveolar echinococcosis of transplantation for incurable alveolar echinococcosis of the Liver:

5 Langnas AN, Sorrell MF. The Budd-Chiari syndrome: a therapeutic gordian knot? Semin Liver Dis 1993; 13: 352-9.

6 Weernink EEM, Huisman AB, Ten Napel CHH. Treatment of Budd-Chiari syndrome by insertion of wall-stent in hepatic vein. Lancet 1991; 338: 644

7 Merkle E, Usadel S, Vogel J, Kern P, Friedrich J-M, Brambs HJ. Alveolar echinococcosis of the Liver - computed tomography findings. Aktuelle Radiol 1995; 5: 101-5. 